\title{
Recognition as a Patient-Centered Medical Home: Fundamental or Incidental?
}

Daniel Doban, $\mathrm{PbD}^{1}$

Mary Honodel McCuistion, $\mathrm{MA}^{2}$

Dominick L. Frosch, $\mathrm{PbD}^{2,3}$

Dorotby Y. Hung, PbD, MA, MPH'

Ming Tai-Seale, $\mathrm{PbD}, \mathrm{MPH} \mathrm{H}^{2}$

${ }^{1}$ Philip R. Lee Institute for Health Policy Studies, University of California San Francisco, San Francisco, California

${ }^{2} \mathrm{Palo}$ Alto Medical Foundation Research Institute, Palo Alto, California

${ }^{3}$ Department of Medicine, University of California Los Angeles, Los Angeles, California

MORE ONLINE

www.annfammed.org

Conflicts of interest: authors report none.

\section{CORRESPONDING AUTHOR}

Ming Tai-Seale, PhD, MPH

Health Policy Research

Palo Alto Medical Foundation Research Institute

Room 446

2350 W El Camino Real

Mountain View, CA 94040-1456

tai-sealem@pamfri.org

\begin{abstract}
PURPOSE Little is known about reasons why a medical group would seek recognition as a patient-centered medical home (PCMH). We examined the motivations for seeking recognition in one group and assessed why the group allowed recognition to lapse 3 years later.
\end{abstract}

METHODS As part of a larger mixed methods case study, we conducted 38 key informant interviews with executives, clinicians, and front-line staff. Interviews were conducted according to a guide that evolved during the project and were audiorecorded and fully transcribed. Transcripts were analyzed and thematically coded.

RESULTS PCMH principles were consistent with the organization's culture and mission, which valued innovation and putting patients first. Motivations for implementing specific PCMH components varied; some components were seen as part of the organization's patient-centered culture, whereas others helped the practice compete in its local market. Informants consistently reported that National Committee for Quality Assurance recognition arose incidentally because of a 1-time incentive from a local group of large employers and because the organization decided to allocate some organizational resources to respond to the complex reporting requirements for about one-half of its clinics.

CONCLUSIONS Becoming patient centered and seeking recognition as such ran along separate but parallel tracks within this organization. As the Affordable Care Act continues to focus attention on primary care redesign, this apparent disconnect should be borne in mind.

Ann Fam Med 2013;11:S14-S18. doi:10.1370/afm.1488.

\section{INTRODUCTION}

T

To paraphrase Casalino, ${ }^{1}$ if a Martian wanted to see patient-centered care, could it safely focus on groups recognized by the National Committee for Quality Assurance (NCQA)? Or might our alien miss practices that truly serve as patient-centered medical homes (PCMHs)? This case study of a group that obtained and then failed to renew recognition can guide our intrepid alien.

PCMH constitutes principles to reshape medical relationships, roles, and responsibilities to bolster primary care and improve outcomes. ${ }^{2-4}$ Defining principles, rather than mandating specific policies or practices, raises challenges of implementation. ${ }^{5}$ Implementing NCQA-defined components can lead to NCQA recognition, but the power of PCMH exists, at least partly, in the idea of a home that serves patients flexibly and humanely. ${ }^{6}$ Practices thus firmly committed to serve as a patient home may weigh the costs and benefits of implementing particular NCQA components. ${ }^{7}$

The medical home may address defects in the US health care system, so health care leaders focus on what makes a home and how to foster and reward "homeness." payment systems and incentives are associated with homelike practices and structures. ${ }^{14,15}$ Demonstration projects provide insights into how to transform a practice into a home and what kinds of environment homes 
need to flourish. ${ }^{16}$ But different practices in diverse environments can and should serve as medical homes. Previous research provides fewer insights into how or why practices may embrace PCMH principles and apply for recognition, or, alternatively, why a "homelike" practice might not seek recognition.

To extend our understanding of how and why a practice becomes a home, we conducted a qualitative case study of a large multispecialty group that achieved NCQA recognition in 10 primary care clinics. We examined the context surrounding PCMH transformation and recognition. As can happen in qualitative studies, emergent issues introduced new analytical foci. ${ }^{17,18}$ During our project, the group decided not to renew PCMH recognition - a decision that raised new questions for the study. Why was the practice letting recognition lapse? Wasn't it important for the practice to be known as a NCQA-recognized PCMH? How had the practice decided to pursue recognition in the first place? Did the decision to let recognition lapse indicate a weakening commitment to patient centeredness? We use data from key informant interviews to examine these questions.

\section{METHODS}

\section{Setting}

We studied a large nonprofit multispecialty group practice in northern California (hereafter referred to simply as the group; per institutional review board protocol, all names are pseudonyms) that serves 4 counties and more than 700,000 patients who mirror Northern California demographically. The group has approximately 4,500 employees and 1,000 physicians, one-half primary care and one-half specialists. Payment mix is $80 \%$ preferred provider organization and $20 \%$ health maintenance organization. Of 17 primary care clinics, 11 were recognized by NCQA in 2007-2010-6 at Level 2 and 5 at Level 3 (the highest rating).

As part of a mixed methods case study of PCMH at the group, a multidisciplinary team of social scientists carried out qualitative research led by 2 ethnographers (D.D., M.H.M.) with extensive qualitative experience. In addition, 2 economists involved in the larger study provided comments, and an additional sociologist participated in coding.

\section{Sample}

We conducted 38 semistructured in-person interviews at practice sites with front-line staff (physicians, nurses, medical assistants) and administrators (department leads, division heads, and senior executives). Participants were chosen purposively to include personnel involved with PCMH implementation across locations and at different organizational levels.

\section{Data Collection}

Interviews took approximately 60 minutes and focused on PCMH implementation. Participants provided written consent but were not compensated. Interviews were audio-recorded and transcribed by a professional service. The interview guide addressed the informant's concept of PCMH, decision to seek and then not renew NCQA recognition, organizational and cultural meaning of recognition, and implementation barriers or facilitators. The guide is given in Supplemental Appendix 1 (available online http://annfammed. org/content/11/Suppl_1/S14/suppl/DC1). One member of the team (M.H.M.) attended all but 2 interviews, and most interviews included a second team member (D.D., D.Y.H., M.T-S., or D.L.F.).

\section{Data Management and Analysis}

All transcripts were entered into ATLAS.ti (ATLAS.ti Scientific Software Development GmbH). Two authors (D.D., M.H.M.) led biweekly meetings to discuss data collection, emergent themes, and sampling strategy. We drafted a codebook to reflect study aims and modified it iteratively based on ongoing analysis. The codebook had 2 tiers: a component code identified particular PCMH components and a substantive code tagged content. We also included codes for cross-cutting themes. Transcripts were coded at the paragraph level. Five interviews were coded by 2 independent coders to establish reliability, and coding differences were discussed and reconciled. When one-half of the interviews had been completed, the team held a daylong retreat to finalize codes, discuss emergent themes, and plan final interviews and analyses that were carried out by the 2 qualitative leads.

\section{RESULTS}

Our interviews shed light on why the group initially obtained NCQA recognition and then decided against renewal. Most informants reported the group valued patient centeredness and acting as a medical home as being consistent with the organization's fundamental values. An incidental opportunity in the local market explained why the group sought and then decided again renewing NCQA recognition.

\section{PCMH Principles and Organizational Values}

Key informants told us that patient centeredness and other key principles of PCMH had always been part of the group's organizational culture. Executives said founders of the group wanted patients to have a "1-stop shop" for primary and specialty care. Nearly all clinic staff agreed with the sentiment a senior administrator expressed: "the idea of the patient at the center I think 
has always been at the core of how this group operates." Informants cited this orientation as responsible for the organization's willingness to experiment with new initiatives and innovations, as a physician-administrator put it, "We want to improve processes so that we take better care of patients." Another physician leader cited a number of care redesign projects_-"a patient care medical home, primary care redesign or innovation center, MyHealth Online [a Web-based patient portal]" — that he felt illustrated how "there's always been attempts to improve care, always." As a nurse summarized: "To me, process improvement and making things better for our patients should be incentive enough."

The organization did not embrace patient centeredness blindly, however, as leaders recognized that "our model of patients first" had to bend to practical constraints and limits. Fee for service was a common concern, as this administrator noted:

The current primary care medical home concept is really an expansion of talent to support the primary care physician, whether it's pharmacy, social work, etcetera, to enable the primary care physician to really have a larger panel with more support and be able to be more focused on medical decision making and have the support needed, in a team delivery concept...[However] it is not cost-effective in a feefor-service environment. In fact, it's unsustainable in a feefor-service environment.

Physicians voiced concerns about PCMH components perceived to be burdensome. One physicianadministrator recalled a plan to expand e-mail so "every single patient could just e-mail the doctor for free." This was among the "worst fears" of physicians who worried that it would "open the floodgates" and to overwhelming demand that would amount to "shooting ourselves in the foot." Fiscal constraints impeded adoption of other patient-centered innovations such as care coordination. Coordination was seen as a benefit for patients, but, as a senior administrator articulated, "how do you build patient-centered medical home when the adjunct people are almost as costly as primary care doctors?" Informants also noted the difficulty of implementing the vague principles of PCMH. In the words of a physician administrator:

How do you incentivize right care? Or how do you incentivize avoiding care, unnecessary care, or investing in nonreimbursed stuff in a fee-for-service environment, even when that nonreimbursed stuff provides value? How do you do that? That's the most important question.

Implementing PCMH components that allowed physicians to see more patients was strategically important. In the compelling image offered by one executive, the group has:
.. a foot in each of 2 canoes, going down the rapids, and the canoes are diverging...The 2 canoes are fee for service and some form of capitation...Ultimately, we will go risk sharing, so that's why we're developing the panel size...it's preparation for the future.

In sum, PCMH recognition was consistent with the group's fundamental values: being patient oriented, innovating to keep up with local competitors, and increasing efficiency in preparation for a future that requires lower total costs.

\section{PCMH Recognition and Incidental Circumstances}

Although the group valued $\mathrm{PCMH}$ principles, it pursued NCQA recognition only after being offered a financial incentive. A physician-administrator explained the reasoning of a group of local employers who put up to $\$ 150,000$ on the table: "'Let's do another type of pay-for-performance program with our local medical groups, and let's make it around IT [information technology] infrastructure because we know that that's associated with better care."' Another physician leader noted that the group responded to the employer offer in part to improve care but also because it offered the chance "to talk to a coalition of large employers, in a committee without contracting any insurance company intermediaries." The employers accepted NCQA recognition as evidence of IT infrastructure and tied the bonus to PCMH level. Even though the employer group bonus "wasn't a huge factor" (midlevel administrator) and "barely covered our costs because this was such a costly thing in terms of people's time" (physician-administrator), the employer challenge still prompted the group to seek recognition.

Recognition per se, was not an organizational priority however. An administrator stated, "If you said we're going to start [a] project, and we're going to improve the way we treat patients, well, I'm all there. That's interesting to me. But I don't really care if there's a certification [sic]." Some administrators were even unaware that the group had received NCQA recognition as a PCMH. For example, after being told by the interviewer that recognition had lapsed, one executive said, "I didn't know that, but okay."

Group leaders involved in applying for recognition recalled "this was a painful process" where it was "not clear exactly what we have that would really fit [NCQA requirements]...we would have roundtables where we would say, 'Okay this is what they're looking for."' No centralized effort was made to seek recognition; each division relied on its own staff and resources. Some divisions made do with fewer resources as this informant recalled proudly: "It doesn't matter if we have less staff than everybody else, it doesn't matter if we have less implementation resources 
than everybody else. We just figure out how to do it and we do it." Nevertheless, without an organizationwide commitment to apply for recognition for all clinics, recognitions were sought for only one-half of the primary care clinics, clinics received different levels of recognition, and higher levels of recognition seemed to go to clinics that were better staffed and resourced.

In 2010, as the NCQA recognition obtained in 2007 was set to expire, the group explored whether payers remained interested in $\mathrm{PCMH}$ recognition. A physician-administrator recalled asking contracting staff, "when you're sitting down with health plans or PPOs [preferred provider organizations] and you're around the bargaining table, is anybody asking about certification (sic)... as a patient-centered medical home? The answer was no." This information was taken into account as group leaders weighed the costs and benefits of recognition against competing organizational priorities, such as strategic planning for redesigning chronic care and an organization-wide integration of disparate scheduling and electronic health record systems into one common system. The absence of enthusiasm from payers and pressing internal competing demands resulted in the decision to let the recognition lapse.

As noted above, contextual factors played a key role in the findings we observed. These factors are summarized in Supplemental Appendix 2 (available online http://annfammed.org/content/11/Suppl_1/ S14/suppl/DC1).

\section{DISCUSSION}

PCMH principles bolster primary care's role, but evaluating and rewarding commitment to these principles is a challenge. This case study builds on insights gleaned from earlier work on PCMH implementation. ${ }^{5}$

$\mathrm{PCMH}$ transformation has generally been seen as a permanent change to make a practice more patient centered-a conversation that is duly recognized. We found, however, that transformation and recognition followed separate and parallel tracks. The group committed to patient-centered principles before PCMH became popular, and that commitment persisted after recognition lapsed. This study suggests that a transformed organization may nevertheless decide to forgo official recognition and thus suggests a need to examine how PCMH is recognized. The group invested in patient centeredness in response to fundamental values and market conditions; it concluded that the stamp of official recognition from NCQA was an unimportant signal to local payers. There appears to be a potential disconnect between PCMH transformation and $\mathrm{PCMH}$ recognition. If a group is not recognized by NCQA, that does not mean the group is not a PCMH.
A second theme that emerged in this study relates to incentives. Previous studies and commentaries have stressed the need to allow adequate time and money to foster transformation. ${ }^{5}$ Some suggest approaching transformation as a multistep process that begins with creation of a receptive environment before attempting the development of a PCMH. This group's experience is consistent with this view; informants stressed the organization's openness and readiness to change and innovate as these processes were felt to be strategic necessities, especially as the organization sought to become more patient centered and better prepared for population management and total cost reduction. On the other hand, NCQA recognition per se was of limited interest. The practice sought recognition when a relatively small incentive was provided and allowed it to lapse when the incentive lapsed. The lapse occurred even as the national policy tide moved in the opposite direction and PCMH was becoming more visible in national health policy discussions. ${ }^{19}$

This case study thus tells a tale of 2 kinds of incentives. On the one hand, the group's $\mathrm{PCMH}$ recognition appears to have been merely a response to an incidental opportunity brought about by an invitation from a local coalition of employers in a highly competitive market for privately insured patients, with a small 1-time monetary reward. On the other hand, the fact that the group was able to respond to that small incentive reflected an organizational readiness that was built on PCMH principles. For fundamental cultural and strategic reasons, the group had committed to or carried out key transformations on the road to $\mathrm{PCMH}$, for example, investing in electronic health records, institutionalizing quality reporting, and overcoming clinician resistance to hot-button PCMH components such as same-day access and free patient e-mails to physicians. With these major transformational hurdles behind it, applying for and obtaining official recognition represented a merely incidental final barrier-a hurdle consisting of paperwork that ultimately did not seem worth clearing.

Our conclusions are limited because this is a single case study and results cannot be generalized and because our data come from informant interviews about past events rather than direct observation. In exploring barriers to and facilitators of PCMH transformation and recognition with key informants, we discovered that $\mathrm{PCMH}$ transformation and recognition are distinct entities. In this case, organizational leaders who believed in the strategic importance of medical home principles, for reasons of patient care as well as business strategy, committed substantial resources to transformation. The group then responded to a 1 -time incentive to achieve recognition. But, given the 
absence of a mandate from payers 3 years later and the multitude of internal competing demands, few in the organization saw advantages in recognition per se.

As the Affordable Care Act continues to focus attention on transforming health care delivery, the differences in incentives we have seen in this case study are worthy of further consideration. At the group, a large incentive-instituting a distinct culture and remaining competitive in its local market-inspired substantial transformation. A small, 1-time financial incentive then sparked a change in labels. With the Affordable Care Act looming on the horizon, payers have begun to rethink reimbursement structures, and private insurers have initiated programs to incentivize providers to offer either components of or a complete $\mathrm{PCMH} .{ }^{20} \mathrm{~A}$ key question will be distinguishing when these programs amount to a "large incentive" that has the potential to be transformational as opposed to a "small incentive" that merely produces a change in labeling. The evidence from this case study suggests that $\mathrm{PCMH}$ recognition per se at times amounts to a small incentive to change labels. This case study cannot speak to what might constitute large incentives powerful enough to change provider behavior in other US health care markets. Returning to the Martian looking for a PCMH on earth, we would recommend that it look beyond the label.

To read or post commentaries in response to this article, see it online at http://www.annfammed.org/content/11/Suppl_1/S14.

Key words: organizational case studies; change, organizational; incentives; motivation; patient-centered medical home; practice-based research

Submitted June 21, 2012; submitted, revised, November 29, 2012; accepted December 10, 2012.

Funding support: This work was supported by grant R18 HS019167 (Tai-Seale, Principal Investigator) from the Agency for Healthcare Research and Quality.

Disclaimer: The content is solely the responsibility of the authors and does not necessarily represent the official views of the Agency for Healthcare Research and Quality.

Acknowledgments: We are grateful to Sukyung Chung, PhD, for coleading some of the interviews. Dr Chung and Hal Luft, PhD, provided valuable comments on previous drafts of this manuscript. Ashley Stone provided valuable editorial support. Finally, we gratefully acknowledge the participation of the key informants who spent time to share their experiences.

\section{References}

1. Casalino LP. Analysis \& commentary. A Martian's prescription for primary care: overhaul the physician's workday. Health Aff (Millwood). 2010;29(5):785-790.

2. Stange KC, Nutting PA, Miller WL, et al. Defining and measuring the patient-centered medical home. J Gen Intern Med. 2010;25(6):601-612.

3. Adams J, Grundy P, Kohn M, Mounib E. Patient-Centered Medical Home: What, Why, and How. Somers, NY: IBM Institute for Business Value; 2009;23.

4. Larson EB, Reid R. The patient-centered medical home movement: why now? JAMA. 2010;303(16):1644-1645.

5. Nutting PA, Miller WL, Crabtree BF, Jaen CR, Stewart EE, Stange KC. Initial lessons from the first national demonstration project on practice transformation to a patient-centered medical home. Ann Fam Med. 2009;7(3):254-260.

6. Fisher ES. Building a medical neighborhood for the medical home. N Engl J Med. 2008;359(12):1202-1205.

7. Kuzel AJ, Skoch EM. Achieving a patient-centered medical home as determined by the NCQA-at what cost, and to what purpose? Ann Fam Med. 2009;7(1):85-86.

8. Kilo CM, Wasson JH. Practice redesign and the patient-centered medical home: history, promises, and challenges. Health Aff (Millwood). 2010;29(5):773-778.

9. Homer CJ, Baron RJ. How to scale up primary care transformation: what we know and what we need to know? J Gen Intern Med. 2010;25(6):625-629.

10. Sia C, Tonniges TF, Osterhus E, Taba S. History of the medical home concept. Pediatrics. 2004;113(5 Suppl):1473-1478.

11. Stange KC, Miller WL, Nutting PA, Crabtree BF, Stewart EE, Jaén CR. Context for understanding the National Demonstration Project and the patient-centered medical home. Ann Fam Med. 2010;8(Suppl 1): S2-S8, S92.

12. Bitton A, Martin C, Landon BE. A nationwide survey of patient centered medical home demonstration projects. J Gen Intern Med. 2010;25(6):584-592.

13. Carrier E, Gourevitch MN, Shah NR. Medical homes: challenges in translating theory into practice. Med Care. 2009;47(7):714-722.

14. Rittenhouse DR, Casalino LP, Gillies RR, Shortell SM, Lau B. Measuring the medical home infrastructure in large medical groups. Health Aff (Millwood). 2008;27(5):1246-1258.

15. Rittenhouse DR, Casalino LP, Shortell SM, et al. Small and mediumsize physician practices use few patient-centered medical home processes. Health Aff (Millwood). 2011;30(8):1575-1584.

16. Crabtree BF, Nutting PA, Miller WL, Stange KC, Stewart EE, Jaén CR. Summary of the National Demonstration Project and recommendations for the patient-centered medical home. Ann Fam Med. 2010;8(Suppl 1):S80-S90, S92.

17. Hoff TJ. Toward a culture of qualitative thinking in health services research. Med Care Res Rev. 2011;68(1):49-55.

18. Weiner BJ, Amick HR, Lund JL, Lee SYD, Hoff TJ. Use of qualitative methods in published health services and management research: a 10-year review. Med Care Res Rev. 2011;68(1):3-33.

19. Iglehart JK. Primary care update-light at the end of the tunnel? N Engl J Med. 2012;366(23):2144-2146.

20. Dentzer S. One payer's attempt to spur primary care doctors to form new medical homes. Health Aff (Millwood). 2012;31(2):341-349. 